Cerenovus, Cerevatech Medical, Inc.,. 4; C; Adona Medical, Inc., Amnis Therapeutics, Bend IT Technologies, Ltd., BlinkTBI, Inc, Buffalo Technology Partners, Inc., Cardinal Consultants, LLC, Cerebrotech Medical Systems, Inc, Cerevatech Medical.

\section{LB-005 THE INSIGHT REGISTRY: A MULTI-CENTER, MULTI-OMIC STROKE CLOT STUDY, PRELIMINARY RESULTS}

1J Fraser*, ${ }^{2}$ V Vicari, ${ }^{3} \mathrm{~A}$ Nanda, ${ }^{4} \mathrm{E}$ Cheng-Ching, ${ }^{5} \mathrm{~K}$ Woodward, ${ }^{2} \mathrm{~B}$ Rapoport, ${ }^{2} \mathrm{C}$ Kellner. ${ }^{1}$ University of Kentucky, Lexington, $\mathrm{KY} ;{ }^{2}$ Icahn School of Medicine at Mount Sinai, New York, NY; ${ }^{3}$ SSM Health, Fenton, MO; ${ }^{4}$ Premier Health - Clinical Neuroscience Institute at Miami Valley Hospital, Dayton, $\mathrm{OH}_{;}{ }^{5}$ Fort Sanders Regional Medical Center, Knoxville, TN

\subsection{6/neurintsurg-2021-SNIS.246}

Introduction Understanding clot composition and associated genomic, epigenomic, and proteomic signatures could provide insight into thrombus biology and etiology, and aid in prognosis in stroke. We report preliminary results in the first 50 subjects enrolled in this multi-omic clot collection registry. Our objective was to report our initial results from the INSIGHT Registry, a multicentered 'multi-omic' analysis of thrombi associated with acute hemorrhagic and ischemic stroke.

Abstracts LB-005 Table 1 Demographics and clinical characteristics

\begin{tabular}{|c|c|c|}
\hline & Ischemic Stroke $(\mathrm{N}=46)$ & $\begin{array}{c}\text { Hemorrhagic Stroke } \\
(\mathrm{N}=4)\end{array}$ \\
\hline Age (yxs) & $67.5(37,92) ; \mathrm{n}=46$ & $63(56,70) ; n=4$ \\
\hline \multicolumn{3}{|l|}{ Sex } \\
\hline Male & $43.5 \%(20 / 46)$ & $50 \%(2 / 4)$ \\
\hline Female & $56.5 \%(26 / 46)$ & $50 \%(2 / 4)$ \\
\hline \multicolumn{3}{|l|}{ Race } \\
\hline Asian & $0.0 \%(0 / 46)$ & $25 \%(1 / 4)$ \\
\hline Black/African American & $13 \%(6 / 46)$ & $0.0 \%(0 / 4)$ \\
\hline White & $73.9 \%(34 / 46)$ & $0.0 \%(0 / 4)$ \\
\hline Multiracial & $2.2 \%(1 / 46)$ & $0.0 \%(0 / 4)$ \\
\hline Other & $10.9 \%(5 / 46)$ & $75 \%(3 / 4)$ \\
\hline \multicolumn{3}{|l|}{ Comorbidities } \\
\hline History of Atrial Fibrillation & $10.9 \%(5 / 46)$ & $0.0 \%(0 / 4)$ \\
\hline Diabetes & $23.9 \%(11 / 46)$ & $25 \%(1 / 4)$ \\
\hline Hypertension & $73.9 \%(34 / 46)$ & $50 \%(2 / 4)$ \\
\hline Hyperlipidemia & $43.5 \%(20 / 46)$ & $50 \%(2 / 4)$ \\
\hline \multicolumn{3}{|l|}{ NIHSS on Admission } \\
\hline Minor stroke (1-4) & $15.2 \%(7 / 46)$ & $25 \%(1 / 4)$ \\
\hline Moderate stroke (5-15) & $41.3 \%(19 / 46)$ & $50 \%(2 / 4)$ \\
\hline Moderate/Severe (16-20) & $13 \%(6 / 46)$ & $0.0 \%(0 / 4)$ \\
\hline Severe stroke $(\geq 21)$ & $30.4 \%(14 / 46)$ & $0.0 \%(0 / 4)$ \\
\hline Missing & $0.0 \%(0 / 4)$ & $25 \%(1 / 4)$ \\
\hline \multicolumn{3}{|l|}{ NIHSS at Discharge } \\
\hline Recovered (0) & $17.4 \%(8 / 46)$ & $0.0 \%(0 / 4)$ \\
\hline Minor stroke (1-4) & $28.3 \%(13 / 46)$ & $0.0 \%(0 / 4)$ \\
\hline Moderate stroke (5-15) & $19.6 \%(9 / 46)$ & $75 \%(3 / 4)$ \\
\hline Moderate/Severe (16-20) & $8.7 \%(4 / 46)$ & $0.0 \%(0 / 4)$ \\
\hline Severe stroke $(\geq 21)$ & $4.3 \%(2 / 46)$ & $25 \%(1 / 4)$ \\
\hline Missing & $21.7 \%(10 / 46)$ & $0.0 \%(0 / 4)$ \\
\hline \multicolumn{3}{|l|}{ TICI Score } \\
\hline TICI $0-2 \mathrm{~A}$ & $4.3 \%(2 / 46)$ & NA \\
\hline TICI 2B & $15.2 \%(7 / 46)$ & NA \\
\hline TICI $2 \mathrm{C}$ & $10.9 \%(5 / 46)$ & NA \\
\hline TICI 3 & $69.6 \%(32 / 46)$ & NA \\
\hline LKW to Recanalization (Minutes) & $310(119,7230) ; \mathrm{n}=42$ & NA \\
\hline
\end{tabular}

Material and Methods Subjects age $\geq 18$ years, treated frontline with the Penumbra System for thrombectomy or the Artemis Neuro Evacuation device for minimally invasive endoscopic intracerebral hematoma evacuation as standard of care are included. Patient demographics, medical history, radiographic, and procedural information are collected in conjunction with extracted clot and concurrent extracranial arterial blood. Upon collection, clots were placed in RNAlater for preservation of protein and RNA and peripheral blood in K2EDTA tubes, allowing sample shipment at ambient temperature. RNA isolated from clot and blood were quality controlled by TapeStation and quantified by QuBit. Protein is quantified by bicinchoninic acid (BCA) assay. Genomic DNA isolated from peripheral blood is sequenced using the Illumina Infinium Global Screening Array. Follow-up clinical assessments occur at discharge and/or day 7, and at 90 days post-procedure.

Results 50 subjects were enrolled from 8 active sites between 02/2021 and 06/2021 (table 1). As expected, most enrollments were in ischemic stroke thrombectomy (46) versus intracranial hemorrhage (4), reflecting the relative incidence and utilization of those procedures. The initial dataset provided reflects realworld results consistent with published thrombectomy studies. RNA isolated from clots had an average RNA integrity score (RIN) of $4( \pm 1)$ ranging from 2-8, while RNA isolated from sytemic arterial blood averaged $5( \pm 2)$ with a range from 2-9 with 1 sample fail $(n=48)$. Total protein isolated from clots averaged 3028ug $( \pm 5407)$ ranging from 32-23,451ug $(n=24)$, while total protein isolated from blood averaged 2026ug $( \pm 1265)$ ranging from $241-5334(n=48)$.

Conclusion These preliminary results demonstrate the realworld applicability of the INSIGHT study to typical stroke thrombectomy population. Furthermore, the tissue processing protocols are providing high-quality RNA and protein for analysis, which is currently underway.

Disclosures J. Fraser: 1; C; Penumbra, University of Kentucky. 2; C; Stream Biomedical, Medtronic. 4; C; Cerelux, Fawkes Biotechnology. J. Vicari: None. A. Nanda: None. E. ChengChing: None. K. Woodward: None. B. Rapoport: None. C. Kellner: None.

\section{LB-006 COLLATERAL IMAGING OF STROKE PATIENTS IN THE LATE WINDOW PRIOR TO ENDOVASCULAR THROMBECTOMY RESULTS FROM A POOLED INDIVIDUAL PATIENT-LEVEL MULTICENTER ANALYSIS}

${ }^{1} \mathrm{M}$ Almekhlafi* ${ }^{2}{ }^{2} \mathrm{~J}$ Thornton, ${ }^{3} \mathrm{I}$ Casetta, ${ }^{1} \mathrm{M}$ Goyal, ${ }^{4} \mathrm{~N}$ Stefania, ${ }^{5} \mathrm{D}$ Herlihy, ${ }^{6} \mathrm{E}$ Fainardi, ${ }^{5} \mathrm{~S}$ Power, ${ }^{7} \mathrm{~V}$ Saia, ${ }^{5} \mathrm{~A}$ Hegarty, ${ }^{8} \mathrm{G}$ Pracucci, ${ }^{1} \mathrm{~A}$ Demchuk, ${ }^{9} \mathrm{~S}$ Mangiafico, ${ }^{5} \mathrm{~K}$ Boyle, ${ }^{4} \mathrm{P}$ Michel, ${ }^{1} \mathrm{~F}$ Bala, ${ }^{1} \mathrm{R}$ Gill, ${ }^{10} \mathrm{~A}$ Kuczynski, ${ }^{1} \mathrm{~A}$ Ademola, ${ }^{1} \mathrm{M}$ Hill, ${ }^{11} \mathrm{D}$ Toni, ${ }^{12} \mathrm{~S}$ Murphy, ${ }^{13} \mathrm{~B}$ Kim, ${ }^{1} \mathrm{~B}$ Menon. ${ }^{1} \mathrm{U}$ of Calgary, Calgary, AB, Canada; ${ }^{2}$ Neuroradiology, Beaumont Hospital, Dublin, Ireland; ${ }^{3}$ Clinica Neurologica, University of Ferrara, Ferrara, Italy; ${ }^{4}$ Lausanne University Hospital, Lausanne, Switzerland; ${ }^{5}$ Beaumont Hospital, Dublin, Ireland; ${ }^{6}$ University of Florence, Florence, Italy; ${ }^{7}$ Santa Corona Hospital, Pietra Ligure (SV), Italy; ${ }^{8}$ Careggi University Hospital, Florence, Italy; ${ }^{9}$ IRCCS Neuromed, Pozzilli (IS), Italy; ${ }^{10} \mathrm{U}$ of Toronto, Toronto, ON, Canada; ${ }^{11}$ Sapienza University Hospital, Rome, Italy; ${ }^{12}$ The Mater Misericordiae University Hospital, Dublin, Ireland; ${ }^{13}$ Seoul National University Bundang Hospital, Seoul, Korea, Republic of

\subsection{6/neurintsurg-2021-SNIS.247}

Background Collateral assessment using CT angiography (CTA) is promising to increase the eligibility for endovascular thrombectomy (EVT) in the late window (6-24 hours). The outcome of these patients compared to those selected using perfusion imaging is not clear. We aimed to describe the 